\section{$\underset{\substack{\text { hommes } \\ \text { \& migrations }}}{ }$}

\section{Hommes \& migrations}

Revue française de référence sur les dynamiques

migratoires

$1317-1318 \mid 2017$

L'Europe en mouvement

\title{
Les migrations de travail intra-européennes entre ruptures et continuités
}

\section{Aurore Flipo}

\section{(2) OpenEdition \\ Journals}

Édition électronique

URL : http://journals.openedition.org/hommesmigrations/3875

DOI : 10.4000/hommesmigrations.3875

ISSN : 2262-3353

Éditeur

Musée national de l'histoire de l'immigration

Édition imprimée

Date de publication : 1 avril 2017

Pagination : 69-77

ISBN : 978-2-919040-38-4

ISSN : $1142-852 X$

Référence électronique

Aurore Flipo, «Les migrations de travail intra-européennes entre ruptures et continuités », Hommes \& migrations [En ligne], 1317-1318 | 2017, mis en ligne le 01 avril 2020, consulté le 03 janvier 2020. URL : http://journals.openedition.org/hommesmigrations/3875; DOI : 10.4000/hommesmigrations.3875 


\title{
LES MIGRATIONS DE TRAVAIL INTRA-EUROPÉENNES ENTRE RUPTURES ET CONTINUITÉS
}

Par AURORE FLIPO, sociologue associée à l'UMR Pacte, université de Grenoble-Alpes.

\author{
Dans un contexte de crise migratoire aux frontières \\ de l'Europe, les migrations intra-européennes, en particulier \\ d'Est en Ouest, continuent de se développer. Entre 2004 \\ et 2014, elles ont concerné près de sept millions de personnes. \\ De nouvelles destinations se sont progressivement affirmées \\ comme le Royaume-Uni ou l'Espagne. Cette mobilité, où le motif \\ économique occupe une part importante mais non exclusive, \\ renforce l'intégration du marché du travail européen tout \\ en maintenant les inégalités sociales au sein de l'Europe entre \\ les populations migrantes et celles de leurs pays d'accueil.
}

Depuis le début des négociations de pré-adhésion des nouveaux États membres d'Europe centrale et orientale à l'Union européenne dans les années 1990, les migrations intra-européennes, et en particulier d'Est en Ouest, ont fait l'objet de nombreuses spéculations politiques. Peu, cependant, se sont vérifiées : si l'exode massif consécutif à la chute de l'URSS n'a pas eu lieu, d'importantes migrations de travail se sont développées dans la première décennie du XXI ${ }^{e}$ siècle. Entre 2004 et 2014, près de quatre millions de citoyens issus des nouveaux États membres se sont installés dans les pays de l'ex-Union européenne à quinze et, selon l'Organisation de coopération et de développement économiques (OCDE), environ sept millions de travailleurs se sont déplacés d'Est en Ouest au cours de la même période. Enfin, si la crise de 2008-
2009 n'a pas provoqué non plus de retour massif des migrants dans leur pays d’origine, elle a réactivé certaines routes migratoires du Sud au Nord (Portugais en France par exemple) et en a créé de nouvelles (Espagnols en Norvège...).

Élevé au rang d'acquis symbolique ou accusé de promouvoir le dumping social et la criminalité, l'espace Schengen est aujourd'hui associé à la liberté de mouvement des travailleurs (voire souvent confondu avec elle), et l'on discute régulièrement de la renégociation ou de la suppression de ce dispositif. Le système de Schengen est pourtant au départ essentiellement un dispositif de coopération judiciaire et policière, ayant pour but de faciliter la circulation des marchandises en Europe par l'effacement des frontières physiques intérieures tout en renforçant les contrôles sur la frontière extérieure de l'Union, et de manière 
déterritorialisée ${ }^{1}$. Cependant, à chaque élargissement, Schengen a fait l'objet d'une " angoisse chronique $^{2}$ » de la part des États membres sur le transfert de souveraineté que nécessite l'existence d'une frontière extérieure commune. Plus récemment, la question des réfugiés a remis en évidence les tensions et les conflits entre les États membres au sujet de la gestion de l'espace commun européen qui en découle.

C'est oublier, cependant, que la mobilité des travailleurs a fait partie de la construction européenne depuis ses premiers balbutiements, bien avant Schengen, dont le premier accord a été signé en 1985. La Communauté économique du charbon et de l'acier est en effet née de l'internationalisation du marché du travail et de l'économie des bassins houillers, situés à cheval sur quatre pays ${ }^{3}$. La libéralisation progressive de la circulation des travailleurs ne s'est cependant jamais faite sans crainte : si, au début des années 2000, l'adhésion de l'Europe centrale et orientale suscite des réticences de la part de l'Europe des quinze, ceétait déjà le cas

La libéralisation progressive de

la circulation des travailleurs

ne s'est cependant jamais faite

sans crainte : si, au début des

années 2000, l'adhésion de

l'Europe centrale et orientale suscite des réticences de la part de l'Europe des quinze, c'était déjà le cas de l'Europe du Sud dans les années 1980. de l'Europe du Sud dans les années 1980.

Par ailleurs, les migrations intra-européennes sont loin d'être réductibles à une simple variable d'ajustement salariale comme l'imaginaient les économistes libéraux. Les indicateurs macro-économiques ne permettent pas d'expliquer ni l'ampleur de l'émigration, ni celle de l'immigration dans les différents pays européens ${ }^{4}$. La mobilité révèle cependant, dans les pays d'origine, les dynamiques sociales et les inégalités face aux marchés du travail locaux, tandis que, dans les pays d'accueil, elle souligne la permanence de la dualité du marché du tra- vail ${ }^{5}$ et la création, au cours des dernières années, de nombreux emplois peu qualifiés et peu rémunérés dans l'industrie et les services.

\section{L'Europe mobile d'hier et d'aujourd'hui}

Si l'on tend à considérer aujourd'hui l'Europe comme l'un des principaux pôles d'immigration dans le monde, il ne faut pas oublier qu'elle a d'abord été marquée d'une part par l'émigration, et d'autre part par une intense mobilité interne. Environ 52 millions de personnes ont ainsi quitté le sol européen pour les Amériques entre 1860 et 19146. Si la tendance s'est rapidement inversée au milieu du XXe siècle, ce n'est pas avant la fin de celui-ci qu'elle s'impose comme un pôle d'immigration majeur comparable à l'Amérique du Nord. La Commission européenne estime ainsi qu'en 2012 l'Union européenne compterait environ 6,5\% de résidents étrangers, dont $37 \%$ sont issus d'un autre État membre de l'Union?.

De même, si les mobilités intra-européennes ont connu une nette accélération depuis le début du XXI ${ }^{\mathrm{e}}$ siècle, elles ne sont toutefois pas un phénomène nouveau. Parallèlement aux migrations vers les Amériques, l'Europe connaît une première période d'intense mobilité dans la seconde moitié du XIX siècle qui, bien que difficile à quantifier avec précisions, concerne plusieurs millions de personnes. ${ }^{8}$. Si l'on s'en tient simplement à létude des recensements contemporains, on constate déjà qu'au début des années 2000 il y avait environ 27 millions de personnes en Europe qui résidaient dans un autre pays que celui où elles étaient nées. Au sein même de l'Union européenne, cela concernait environ 16 millions de personnes ${ }^{9}$. À létude des

2. Ruben Zaiotti, Cultures of Border Control. Schengen and the Evolution of European Frontiers, Chicago, Chicago University Press, 2011. 3. René Leboutte, "Des “travailleurs étrangers” aux “citoyens européens". Mobilité et migrations dans les bassins industriels aux XIXe-XXe siècles ", in Espace populations sociétés, vol 19, n 3, 2001, pp. 243-258. 4. Aurore Flipo, "Les nouvelles migrations de travail intra-européennes. Jeunes Polonais et Roumains au Royaume-Uni et en Espagne ", thèse de doctorat en sociologie, Sciences Po, 2014. 5. Michael J. Piore, Birds of Passage: Migrant Labor and Industrial Societies, Cambridge, Cambridge University Press, 1980. 6. Leslie P. Moch, Moving Europeans: Migration in Western Europe since 1650, Bloomington, Indiana University Press, 1992. 7. Voir le Rapport annuel de la Commission européenne sur l'asile et l'immigration. 8. Philippe Rygiel, Le temps des migrations blanches. Migrer en Occident du milieu du XIXe siècle au milieu du XX siècle, Paris Publibook, 2010 [en ligne]. 9. On se réfère ici aux personnes nées dans l'un des pays membre de l'Union européenne, mais résidant dans un autre. 
chiffres de la population née à l'étranger pays par pays, on constate que les mobilités de voisinage ont toujours été importantes en Europe. Si l'on inclut le Maghreb et la Turquie, qui constituent les rives est et sud de la Mare Nostrum, il apparaît alors que le continent européen est marqué par une multitude de migrations de courte distance, les migrations de longue distance étant essentiellement celles de l'exode vers l'Amérique et de la colonisation. L'étude des mouvements de population intra-européens montre donc que l'intégration régionale (économique et politique) était déjà, bien avant l'Union européenne, pourvoyeuse de migrations. L'existence d'une Scandinavie, d'une Mitteleuropa ou l'héritage de la présence russe dans les pays de la Baltique sont ainsi particulièrement nets lorsque l'on s'intéresse aux recensements nationaux européens. Cependant, la facilitation administrative de la circulation au sein de l'Union européenne, l'accessibilité des nouveaux modes de transport internationaux et la mise en place de politiques visant à encourager la mobilité ont contribué à faire émerger de nouvelles pratiques migratoires intra-européennes.

\section{Le renouveau de l'axe migratoire Est-Ouest}

À partir des années 1990, l'Europe a renoué avec une mobilité Est-Ouest qui avait été partiellement éclipsée pendant les années du rideau de fer. Celleci n'a cependant jamais cessé totalement. En vertu de la politique de rapatriement mise en place par le gouvernement de la République fédérale d'Allemagne et accordant la nationalité aux « Allemands ethniques » résidant à l'Est de la ligne Oder-Neisse, environ 4,5 millions de personnes ont rejoint l'Allemagne entre 1950 et $1990^{10}$, majoritairement en provenance de Pologne, de Roumanie et de l'URSS, aboutissant à l'établissement d'importants réseaux migratoires de part et d'autre du rideau de fer ${ }^{11}$. Parallèlement, des migrations dites " économiques » ont commencé à se structurer dans les années 1980 et 1990 en Europe centrale et orientale, d'abord de manière complémentaire aux flux de rapatriés, puis les remplaçant après 1990. Leurs destinations principales étaient l'Allemagne et l'Autriche. Ces migrations étaient fréquemment de court terme, voire pendulaires, surtout avec le voisin polonais mais aussi depuis les pays d'Europe À partir des années 1990, du Sud-Est, aboutissant l'Europe a renoué avec une au concept de migration mobilité Est-Ouest qui avait " circulaire » ou " incom- été partiellement éclipsée plète $»^{12}$, c'est-à-dire que le foyer principal (aussi bien en termes économiques, au pendant les années du rideau de fer. Celle-ci n'a cependant jamais cessé totalement. sens de l'endroit où l'argent est dépensé, qu'en termes humains, au sens de l'unité familiale) est maintenu dans le pays d'origine, les migrants circulant entre les deux pays. C'est d'abord par le biais des visas de tourisme que ces migrations se sont organisées pendant les années 1990. Dès cette période, les routes migratoires d'Est en Ouest se diversifient : bien que l'Allemagne reste la destination principale, l'Angleterre, l'Espagne, l'Italie voient arriver les premiers migrants est-européens. Certaines minorités sont pionnières dans ces nouveaux mouvements migratoires, à l'instar des communautés adventistes roumaines en Espagne. Cependant, la domination de l'Allemagne sur ce paysage migratoire est sans appel : si l'on tient compte des rapatriés, l'Allemagne concentre alors 61 \% de l'ensemble des Est-Européens vivant en Europe occidentale recensés au début des années 2000. Cet état de fait s'est modifié de manière spectaculaire après l'élargissement : en 2010, d'après les données migratoires nationales, la destination la plus commune est devenue le Royaume-Uni, suivi de l'Espagne et de l'Italie. 


\section{L'intensification des migrations en Europe}

Parallèlement à la modification des destinations migratoires, et bien que les données disponibles posent des problèmes de comparabilitée ${ }^{13}$, on constate, au cours des années 2000, une intensification des migrations au sein de l'espace européen, quelles que soient les sources utilisées. Dans la plupart des pays européens, la mobilité s'est accentuée, que ce soit par l'immigration, l'émigration ou, plus fréquemment, les deux. En Europe de l'Ouest, l'immigration totale a augmenté de $50 \%$ en moyenne sur la période 2000-2010 ${ }^{14}$. Mais ces moyennes cachent d'importantes différences, puisque l'essentiel de cette croissance a été enregistré en Europe du Sud et en Irlande, dans des pays qui n'étaient pas des destinations migratoires jusqu'à récemment, mais

Cependant, tous les pays plutôt des terres d'émigraeuropéens nesont pas tion. La donne change cepenimpliqués de la même manière dans cette intensification des mouvements intra-européens.

Non seulement, le degré auquel ils y participent est très différent, mais encore le type de mobilité (immigration ou émigration, mouvements transfrontaliers, migrations

saisonnières, migrations d'installation) diverge. Enfin, alors que la période a plutôt été caractérisée par une forte émigration en Europe centrale et orientale, l'immigration s'est également développée dans ces pays. Celle-ci émane à la fois des voisins européens, mais aussi du Pakistan, de la Chine et du Vietnam, par exemple. La Hongrie, la Répu- blique tchèque ou encore la Slovénie arborent ainsi des soldes migratoires positifs.

Parallèlement à l'intensification globale de la mobilité, c'est en réalité la mobilité intra-européenne qui a connu l'augmentation la plus forte. Le nombre de citoyens issus des nouveaux États membres résidant dans l'ex-Europe des quinze a été multiplié par quatre entre 2000 et $2012^{15}$. En outre, les pays qui ont connu la plus forte croissance du nombre de résidents étrangers sont ceux dans lesquels la mobilité intra-européenne a le plus augmenté. À titre d'exemple, le Royaume-Uni a connu une hausse de son immigration globale de l'ordre de $30 \%$ entre 2000 et 2010, mais le nombre d'Européens a été multiplié par plus de $7^{16}$; en Irlande, l'immigration totale a presque doublé mais le nombre d'Européens a été multiplié par $12^{17}$.

\section{La diversité des profils nationaux}

Cependant, tous les pays européens ne sont pas impliqués de la même manière dans cette intensification des mouvements intra-européens. Non seulement, le degré auquel ils y participent est très différent, mais encore le type de mobilité (immigration ou émigration, mouvements transfrontaliers, migrations saisonnières, migrations d'installation) diverge. Dans ce vaste tableau des mobilités européennes, il est possible d'entrevoir un certain nombre de changements importants. Tout d'abord, les pays de l'Europe du Sud s'affirment dans cette décennie comme de grands pays d'immigration : l'Espagne, l'Italie et, dans une moindre mesure, la Grèce sont devenus des pays d'immigration majeurs en Europe, et ce malgré la reprise de l'émigration dans la décennie suivante. Ensuite, on pourra relever la prévalence relativement nouvelle du Royaume-Uni comme destination la plus commune pour les migrants intra-européens, en particulier issus des nouveaux États membres de lélar- 
gissement de $2004^{18}$. Bien qu'intégré de manière incomplète à l'Union européenne (et bientôt séparé d'elle ${ }^{19}$ ), le Royaume-Uni constitue un pôle migratoire majeur dans l'espace européen.

L'Allemagne, qui tenait par le passé ce rôle vis-à-vis de l'Europe de l'Est, a occupé une place paradoxale dans cette période : hostile en premier lieu à une ouverture de son marché du travail par peur d'une immigration massive, elle a tout de même enregistré d'importants flux de travailleurs européens, mais sans hausse importante du point de vue du nombre de résidents étrangers jusqu'aux années 2010-2011, date à partir de laquelle les migrations en direction de l'Allemagne augmentent à nouveau, en partie en raison de la crise dans le Sud de l'Europe. Comme l'Autriche, l'Allemagne semble avoir été davantage impliquée dans les migrations frontalières et de court terme, ainsi que dans le «travail détaché20 ».

Par ailleurs, si les profils d'immigration ne sont pas homogènes, les profils d'émigration ne le sont pas non plus. Le poids de la Pologne et de la Roumanie est particulièrement important dans les flux Est-Ouest (75 \% des migrants ${ }^{21}$ ), d'abord pour des raisons démographiques puisqu'il s'agit des deux pays les plus peuplés d'Europe centrale et orientale. Les degrés et les rythmes d'émigration sont toutefois très différents en fonction des pays, et ces différences ont eu plutôt tendance à s'accentuer quà se lisser au cours de la période, en dépit de l'homogénéisation des régimes légaux par le biais de la levée progressive des dispositions transitoires. Ainsi, la Bulgarie et la Roumanie ont des taux d'émigration très importants et en croissance rapide au début des années 2000, tandis que la Hongrie, la Slovénie et la République tchèque ont connu une émigration relativement faible, bien que notable dans certains segments de la population (en particulier les jeunes).

\section{Des mobilités multiples}

Dans certains cas également, la confrontation des données de flux (entrées annuelles de travailleurs étrangers) et de stock (résidents étrangers) évoque l'existence de pratiques migratoires distinctes en fonction des pays ${ }^{22}$. La Hongrie, par exemple, est davantage impliquée dans les migrations pendulaires et transfrontalières avec l'Autriche voisine, qui constitue la principale destination pour les migrants hongrois.

Par ailleurs, la mobilité intra-européenne ne se limite pas à la mobilité Est-Ouest : la mobilité entre les pays «fondateurs » de l'Union européenne s'est également accentuée au cours des années $2000^{23}$. De même, il existe d'importantes mobilités EstEst. Ainsi, l'essentiel des entrées en provenance de l'Union européenne dans les nouveaux États membres provient d'autres pays d'Europe centrale et orientale.

Enfin, la libre-circulation n'est pas la seule forme de mobilité des travailleurs possible au sein de l'Union européenne. Avant lélargissement et même après, d'autres formes de gestion de la mobilité internationale ont pu coexister et contribuer à l'intensification de la mobilité intra-européenne. C'est le cas, par exemple, des accords bilatéraux de main-d'œuvre ${ }^{24}$ ou encore du détachement de travailleurs.

\section{Deux cas exemplaires : Polonais au Royaume-Uni et Roumains en Espagne}

Parmi les nouvelles mobilités ayant émergé au début de ce siècle, les migrations entre la Pologne

19. Le Brexit, soit la sortie du Royaume-Uni de l’Union européenne, a été voté par référendum en juin 2016. La campagne des pro-Brexit s'est appuyée en grande partie sur la critique de la libre-circulation des travailleurs en Europe pour justifier la sortie de l'Union. Voir notamment Aurore Flipo, "La question migratoire au cœur du référendum du Brexit ", in The Conversation, 20 juin 2016 [en ligne]. 20. Le travail détaché permet à des prestataires de services d'utiliser leur propre main-d'œuvre sur le sol d'un autre État membre, sans que celle-ci soit soumise au droit du travail local, à condition que ce détachement soit temporaire. Voir ec.europa.eu. 21. OCDE, Migration for Employment: Bilateral Agreements at a Crossroads, Paris, OECD Publishing, 2004.

22. Bien qu'il ne faille pas perdre de vue que ce sont des données produites différemment et qui ne sont donc pas strictement comparables. 23. Ettore Recchi, "Cross-state mobility in the EU: Trends, puzzles and consequences », in European Societies, vol. 10, $n^{\circ}$ 2, 2008, pp. 197-224. 24. OCDE, Migration for Employment, op. cit. 
et le Royaume-Uni d'une part, et entre la Roumanie et l'Espagne d'autre part, se sont révélées comme étant parmi les plus importantes, impliquant plus d'un million de personnes chacune. La rapidité de ces nouveaux flux migratoires, leur importance quantitative et leurs particularités justifient que l'on s'intéresse de plus près à ces configurations migratoires, en de nombreux points comparables. En 2010, les Polonais représentaient $9 \%$ de l'ensemble des résidents nés à l'étranger au Royaume-Uni, tandis que les Roumains représentaient $10 \%$ de la population étrangère en Espagne. On constate, tout d'abord, que ces flux migratoires se sont constitués dans le contexte d'une augmentation globale et forte de l'immigration dans les pays d'accueil : le nombre de résidents nés à l'étranger augmente de $39 \%$ entre les recensements de 2001 et de 2011 au Royaume-Uni, quand il triple en Espagne. Dans les deux cas, l'essentiel des mouvements migratoires a eu lieu au début des années 2000 , selon des temporalités qui correspondent à des politiques migratoires particulières.

Le Royaume-Uni a été l'un des seuls pays, avec la Suède, à ouvrir pleinement son marché du travail aux ressortissants des nouveaux États membres de l'Union européenne dès le $1^{\text {er }}$ mai 2004. Cependant, cette ouverture fait suite à divers programmes de recrutement de main-d'œuvre esteuropéenne, en particulier dans l'agriculture et l'industrie (Agricultural Workers Scheme et Sector Based Scheme) mais également pour les indépendants, déjà libres de s'installer depuis la fin des années 1990. Dans le cas espagnol, les premiers mouvements migratoires depuis la Roumanie apparaissent également à la fin des années $1990^{25}$, mais c'est en 2002 qu'ils s'accélèrent, consécutivement à la suppression de l'obligation de visa pour les courts séjours, qui permet à de nombreux Roumains de s'insérer dans un marché du travail espagnol marqué par l'importance du travail non déclaré. Parallèlement, depuis 1993, des politiques de quotas étaient appliquées à l'entrée de travailleurs étrangers, ainsi qu'aux résidents jusqu'en 2002, devenant alors un outil de régularisation. À partir de 2002, les permis de travail furent accordés en priorité aux travailleurs issus de pays avec lesquels l'Espagne avait signé des accords bilatéraux, parmi lesquels la Roumanie, la Pologne et la Bulgarie. L'essentiel des mouvements migratoires ayant eu lieu en Espagne entre 2002 et 2007, la migration roumaine fut principalement irrégulière jusqu'à la vague de régularisation de 2005 et l'élargissement de 2007. De 2007 à 2009, les Roumains se sont trouvés dans la situation paradoxale d'avoir la liberté de résider en Espagne, sans avoir pour autant accès pleinement au marché du travail. Enfin, en 2009, la levée des dispositions transitoires a eu pour effet de régulariser de facto l'ensemble de la population roumaine déjà présente sur le territoire.

\section{Qui sont les émigrants?}

Du côté des pays d'accueil, on voit donc que les migrations intra-européennes se sont produites dans le contexte d'une hausse générale de l'immigration de travail.

Du côté des pays d'origine, l'analyse des intentions d'émigration illustre de manière frappante la restructuration des inégalités intra-nationales dans le contexte de la transition post-communiste. Ainsi, l'émigration a touché en particulier les catégories sociales les plus affectées par la flexibilisation et la libéralisation ; en particulier les jeunes qui ne parviennent pas à s'insérer sur un marché du travail de plus en plus compétitif et à s'installer en raison des salaires bas et d'un coût de la vie qui augmente (en particulier les loyers urbains).

Selon les données issues du Diagnostic social polo-

25. Miguel Pajares Alonso, "Comunidades inmigradas de la Europa del Este. El caso del colectivo rumano en Espana ", in Revista Cidob d'Afers Internacionals, $n^{\circ} 84,2008$, pp. 65-79. 26. Le Diagnostic social est une enquête conduite tous les 2 ans depuis 2003 par l'Université des finances et de la gestion de Varsovie. Nous avons utilisé les données issues de la vague de 2007 , qui portent sur un échantillon représentatif de 5532 ménages et 12641 individus. 27. Le Baromètre de l'opinion publique est une enquête annuelle conduite de 1994 à 2007 par la fondation Soros. Nous avons utilisé les données de 2007 qui portent sur un échantillon représentatif de 2000 individus. 
nais ${ }^{26}$ et du Baromètre de l'opinion publique roumain ${ }^{27}$ datant de 2007 et contenant une question sur les intentions de partir à l'étranger pour y travailler, un peu plus d'une personne sur dix, dans ces pays, exprimait un désir de partir ( $11 \%$ en Pologne et $14 \%$ en Roumanie). L'incidence des intentions d'émigrer était particulièrement forte chez les jeunes : un jeune de moins de 25 ans sur trois déclarait, dans les deux pays, avoir l'intention de chercher du travail à l'étranger. C'est dans les niveaux moyens d'éducation (éducation secondaire) que l'on trouve l'incidence la plus forte des désirs d'émigration, mais les étudiants sont également très nombreux à vouloir s'expatrier. Dans les pays d'accueil, on relève en effet un grand nombre de jeunes partis une fois leurs études terminées ou ayant interrompu celles-ci ; tandis qu'un certain nombre de jeunes diplômés du baccalauréat partent dans l'espoir de financer des études supérieures au pays.

Les inégalités ethniques s'expriment dans les intentions d'émigrer en Roumanie puisque $27 \%$ des Roms interrogés ont exprimé le désir de partir : c'est le double du taux observé chez les Roumains de catégorie ethnique déclarée "Roumain ». Enfin, si le niveau de revenu brut ne semble pas influer sur les intentions d'émigrer, la structure des revenus montre de grandes différences. Ainsi, une proportion significativement plus importante des candidats à l'émigration tire ses revenus hors du marché du travail : la solidarité familiale, les transferts sociaux ou l'argent envoyé depuis l'étranger, indiquant, en particulier chez les moins de 35 ans, une faible indépendance financière. Les chômeurs sont également fortement surreprésentés dans les intentions d'émigrer, ainsi que les travailleurs les moins qualifiés et les travailleurs déclassés, c'està-dire ceux pour qui le lien entre revenu et éducation est plus faible.

Ainsi, toutes choses égales par ailleurs, le fait d'être jeune et sans emploi constitue dans les deux pays le facteur le plus influent sur la probabilité de vouloir quitter le pays. Par ailleurs, au niveau macrosocial, on observe une corrélation entre niveau de protection sociale et taux d'émigration ${ }^{28}$. C'est donc bien la précarité qui pousse les jeunes migrants à partir pour l'étranger, la majorité d'entre eux partant avec des projets de court terme amenés plus tard à se modifier.

\section{Des pratiques migratoires diversifiées}

Malgré l'importance de l'enjeu de l'insertion professionnelle dans ces nouvelles migrations, il serait toutefois inapproprié de les réduire au seul prisme économique ${ }^{29}$. La libéralisation des conditions de circulation a en effet permis à la mobilité internationale d'être à la portée d'un plus grand nombre de personnes. En conséquence, on constate une diversité sociale importante parmi les migrants, et les pratiques de mobilité elles-mêmes sont également de plus en plus diversifiées. La migration de travail, qui se caractérise par le fait de prendre un emploi à l'étranger, de manière temporaire ou durable, constitue une pratique de mobilité parmi d'autres et, comme toute pratique, elle varie dans ses formes, ses usages et ses usagers. Si, pour certains groupes sociaux particulièrement fragilisés, la mobilité est une nécessité qui relève de la survie, pour les jeunes issus de milieux plus favorisés, il s'agit avant tout d'une expérience, d'une aventure destinée à sceller le passage à l'âge adulte. Dans les classes moyennes, la mobilité peut constituer un moyen de résister au déclassement par l'acquisition de compétences internationales. Enfin, la mobilité peut être motivée par la recherche d'une meilleure qualité de vie ou par des considérations d'ordre politique.

Enfin, l'appartenance sociale des individus conditionne également l'effet de la mobilité sur les trajectoires. Ainsi, la capacité à capter les opportunités dépend de l'importance des ressources internatio- 
nales préexistantes (réseau social, aisance dans les situations interculturelles, maîtrise des langues étrangères), qui influencent de manière déterminante les possibilités d'intégration socio-professionnelle dans le pays d'accueil.

\section{La mobilité dans le contexte de la libre-circulation}

Si les pratiques migratoires sont de plus en plus diverses, l'étude de l'insertion professionnelle des Polonais et des Roumains montre de son côté de grandes tendances plutôt stables. Les secteurs professionnels dans lesquels les migrants intra-européens se sont insérés ne semblent guère différents des secteurs traditionnellement occupés par les immigrants. Plus encore, l'ouverture du marché du travail aux ressortissants des nouveaux États membres a été conçue, dans le cas de l'Espagne et du Royaume-Uni, dans

L'analyse comparée des flux migratoires intra-européens esquissée ici permet de mettre

en évidence les facteurs

systémiques qui façonnent

le système migratoire

européen contemporain. En

premier lieu, on retiendra

l'augmentation générale et importante de la mobilité des travailleurs au sein de l'espace

européen sur une période relativement courte, allant de la fin des années 1990 à la crise de 2008-2009. la continuité des politiques migratoires sectorielles précédentes. En ce sens, la mobilité européenne « libre » ne l'est pas tant que cela, et le recours à la main-d'œuvre étrangère comporte des stratégies de captation de cette main-d'œuvre, qui semblent pour le moins efficaces au vu du degré de ségrégation professionnelle observable dans les deux pays.

Ainsi, malgré la diversité des niveaux d'éducation observables dans la population migrante, un peu plus d'un tiers des Polonais au Royaume-Uni et près de $40 \%$ des Roumains en Espagne sont employés dans des "professions élémentaires $^{30}{ }^{\prime}$. Cela s'explique, en premier lieu, par la structure des secteurs dans lesquels ils travaillent: en effet, les migrants se trouvent concentrés dans un très petit nombre de secteurs, ceux-ci étant par ailleurs de gros consommateurs de main-d'œuvre étrangère. Au Royaume-Uni, il s'agit principalement de la construction, de la logistique, de l'industrie agro-alimentaire et des services (hôtels, restaurants et nettoyage). En Espagne, il s'agit également de la construction, de l'agriculture et des activités domestiques (aides à domicile, personnel de ménage).

Ainsi, les cadres sociaux qui conditionnent la mobilité du travail ne semblent pas avoir été fondamentalement transformés par la mise en place du droit de circulation des travailleurs, et la ségrégation demeure forte.

\section{Conclusion : I'Europe mobile aujourd'hui}

L'analyse comparée des flux migratoires intra-européens esquissée ici permet de mettre en évidence les facteurs systémiques qui façonnent le système migratoire européen contemporain. En premier lieu, on retiendra l'augmentation générale et importante de la mobilité des travailleurs au sein de l'espace européen sur une période relativement courte, allant de la fin des années 1990 à la crise de 2008-2009. Il ne semble pas exagéré de parler d'une reprise massive de l'immigration de travail durant cette période. Entre 2009 et 2011, on observe une certaine stagnation des mouvements de travailleurs au sein de l'Union, qui reprennent toutefois à partir de 2012.

Les cas particuliers du Royaume-Uni et de l'Espagne, représentant deux configurations nationales différentes, apportent un éclairage sur l'articulation entre le cadre national et le phénomène transnational qu'est la migration. En Espagne, où la migration s'est produite par des voies essentiellement irrégulières, la dépendance aux cycles économiques s'est trouvée renforcée. Les effets de la crise sur l'emploi des migrants ont été bien plus importants qu'au Royaume-Uni, et l'an- 
crage des immigrés dans l'économie souterraine a perduré bien après la levée des restrictions. Au Royaume-Uni, la situation des migrants sur le marché de l'emploi est globalement meilleure, mais les emplois sont souvent de faible qualité et, en raison du niveau de diplôme globalement plus élevé des Polonais, le déclassement est plus fréquent. En outre, la montée de la xénophobie anti-Européens, au lendemain du Brexit, fragilise plus que jamais la position des Polonais dans le pays.

Enfin, du côté des pays de "départ » (bien que cela s'entende, encore une fois, relativement à l'espace intra-européen qui nous intéresse ici, car de nombreux pays de « départ » sont aussi des pays d'arrivée pour d'autres migrants), les dynamiques migratoires témoignent également de l'existence de transformations sociales importantes, liées à l'État-providence et au marché du travail. Au début des années 2000, en Pologne et en Roumanie, les jeunes et les chômeurs étaient les plus susceptibles d'émigrer, bien que les pratiques migratoires touchent un ensemble beaucoup plus hétérogène de la population. Ainsi, l'analyse des désirs d'émigration permet d'apprécier la position différentielle des individus vis-à-vis du principal système d'allocation des ressources de la société marchande : le marché du travail.

L'intégration européenne, d'une part, et l'existence d'inégalités importantes, d'autre part, suscitent la mobilité : ce principe n'est pas nouveau, et la conjonction entre intégration économique et inégalités caractérise le monde globalisé dans son ensemble. Toutefois, cela n'est que le cadre général dans lequel de multiples phénomènes plus complexes s'entrecroisent, comme l'illustre le cas des mobilités intra-européennes. 